\title{
General model of vertical distribution of stars in the Milky Way using complete Jeans equations
}

\author{
Suchira Sarkar^ and Chanda J. Jog ^ \\ Department of Physics, Indian Institute of Science, Bangalore-560012, India
}

Accepted 2019 December 7. Received 2019 December 6; in original form 2019 November 19

\begin{abstract}
The self-consistent vertical density distribution in a thin, isothermal disc is typically given by a sech ${ }^{2}$ law, as shown in the classic work by Spitzer. This is obtained assuming that the radial and vertical motions are decoupled and only the vertical term is used in the Poisson equation. We argue that in the region of low density as in the outer disc this treatment is no longer valid. We develop a general, complete model that includes both radial and vertical terms in the Poisson equation and write these in terms of the full radial and vertical Jeans equations which take account of the non-flat observed rotation curve, the random motions, and the cross term that indicates the tilted stellar velocity ellipsoid. We apply it to the Milky Way and show that these additional effects change the resulting density distribution significantly, such that the mid-plane density is higher and the disc thickness (HWHM) is lower by $30-40$ percent in the outer Galaxy. Further, the vertical distribution is no longer given as a sech ${ }^{2}$ function even for an isothermal case. These predicted differences are now within the verification limit of new, high-resolution data for example from Gaia and hence could be confirmed.
\end{abstract}

Key words: Galaxy: disc-Galaxy: kinematics and dynamics-solar neighbourhoodGalaxy: structure.

\section{INTRODUCTION}

The vertical structure of the stellar disc in a thin galactic disc such as the Milky Way is typically obtained by treating it as an isothermal, self-gravitating system whose density distribution along the vertical direction is given by a sech ${ }^{2}$ form, see the classic work by Spitzer (1942). The disc is treated to be thin and axisymmetric; and a cylindrical coordinate system is used. Hence, the Poisson equation contains only the vertical term, while the radial and azimuthal terms are dropped. Combining it with the vertical Jeans equation or the equation of hydrostatic equilibrium, gives the following equation

$\frac{\partial}{\partial z}\left[\frac{1}{\rho} \frac{\partial\left(\rho \overline{v_{\mathrm{z}}^{2}}\right)}{\partial z}\right]=-4 \pi G \rho$,

where $\rho$ is the density and $\overline{v_{\mathrm{z}}^{2}}$ is the square of the isothermal vertical velocity dispersion. The solution of this equation gives the vertical density distribution:

$\rho(z)=\rho_{0} \operatorname{sech}^{2}\left(z / z_{0}\right)$

where $\rho_{0}$ is the mid-plane density and $z_{0}$ is a measure of scale height. We will refer to this as the sech ${ }^{2}$ model in rest of the paper. A similar treatment is used to study the related problem of the total dynamical mid-plane density or the Oort limit (Oort 1932; Bahcall

^E-mail: suchira@iisc.ac.in (SS); cjjog@iisc.ac.in (CJJ)
1984). Thus, usually in galactic dynamics, the $R$ and $z$ motions are effectively taken to be decoupled. This allows for a simple treatment of the orbits in the plane and those normal to the plane as being decoupled (Mihalas \& Routly 1968; Binney \& Tremaine 1987).

We point out that in the general case, such as when the disc density is low as in the outer parts of the disc or at regions away from the mid-plane, or as in the thick disc, the neglect of the radial term is not justified because in such regions this term may become comparable to the vertical term in the Poisson equation. Hence even for an isothermal case, the resulting density distribution would be different from the standard $\operatorname{sech}^{2}$ distribution.

The radial term in the Poisson equation was indeed included, and for simplicity was obtained using the observed rotation curve, in some earlier papers (Narayan, Saha \& Jog 2005; Banerjee et al. 2011; Sarkar \& Jog 2019). Interestingly, in case of an observed flat rotation curve, the contribution of this term turns out to be identically zero (Narayan et al. 2005) in the mid-plane. Here, instead we use the complete radial Jeans equation to calculate the radial term in the Poisson equation.

Further, in writing both the vertical and radial Jeans equations that are used to obtain the vertical and radial terms of the Poisson equation respectively, typically a simplification is made, namely the so-called cross term $\overline{v_{\mathrm{R}} v_{\mathrm{z}}}$, where the average is taken over the velocity dispersions, is ignored. The term $\overline{v_{R} v_{z}}$ is a component of the velocity ellipsoid tensor whose value is set by the tilt of the velocity ellipsoid with respect to the disc plane, and it also 
represents the coupling between radial and vertical motions. But its contribution has been neglected in most of the dynamical models so far (Bahcall 1984; Cappellari 2008) as is justified for a region very close to the mid-plane in a thin disc. Note that recent kinematic data from observations like radial velocity experiment, sloan digital sky survey, and Gaia show that the stellar velocity ellipsoid is indeed tilted in the meridional plane both in the stellar disc (Hagen et al. 2019; Everall et al. 2019) and in the outer stellar halo (Wegg, Gerhard \& Bieth 2019). It is observed to have an orientation such that it tends to align with a spherical polar coordinate system centred at the centre of the Galaxy. This motivates us to consider this cross term in the Jeans equations.

Here, we solve for the self-consistent vertical distribution of stars using the complete Poisson equation, where the vertical and radial terms are given in terms of complete vertical and radial Jeans equations without the usual simplifications - thus we can consider this as the complete and general model. We calculate the mid-plane density value and scale height of the disc, measured in terms of the half-width at half-maximum (HWHM) of the vertical density distribution, and compare them with the corresponding results obtained from a simple, stars-alone case ( $\mathrm{sech}^{2}$ model) as obtained from our earlier work (Sarkar \& Jog 2018). With the advent of accurate data, as from Gaia or the large sky area multi-object fiber spectroscopic telescope (LAMOST), it is feasible to check such modified density distribution with the observations, hence our study is timely. Some earlier studies have used the full set of Jeans equations to determine vertical force field, shape of the dark matter halo, and local estimate of dark matter density (Bovy \& Tremaine 2012; Hagen \& Helmi 2018; Sánchez-Salcedo, Flynn \& de Diego 2016; Wegg et al. 2019), but the effect on the vertical density distribution of stars has not been studied.

The outline of the paper is as follows - we describe the derivation of the equations along with the input parameters in Section 2, give the results in Section 3, and give the conclusions in Section 4.

\section{FORMULATION OF THE PROBLEM}

\subsection{Derivation of the equations}

Here, we use the full Poisson equation containing both the vertical and radial terms, where the vertical and radial gradients of potential are obtained using the full Jeans equations. We consider the disc to be in a steady-state system and for such a system the Poisson equation is given by

$$
\frac{1}{R} \frac{\partial}{\partial R}\left(R \frac{\partial \Phi}{\partial R}\right)+\frac{\partial^{2} \Phi}{\partial z^{2}}=4 \pi G \rho(R, z)
$$

where $\Phi$ is the gravitational potential, and the terms on the lefthand side denote the radial and vertical terms of the Poisson equation respectively. To calculate the radial and vertical gradients of the potential, we use the radial and vertical axisymmetric Jeans equations in cylindrical coordinates, given as (see Binney \& Tremaine 1987):

$$
\begin{aligned}
& \frac{\partial \Phi}{\partial R}=-\frac{1}{\rho} \frac{\partial}{\partial R}\left(\rho \overline{v_{\mathrm{R}}^{2}}\right)-\frac{1}{\rho} \frac{\partial}{\partial z}\left(\rho \overline{v_{\mathrm{R}} v_{\mathrm{z}}}\right)-\frac{\left(\overline{v_{\mathrm{R}}^{2}}-\overline{v_{\phi}^{2}}\right)}{R}, \\
& \frac{\partial \Phi}{\partial z}=-\frac{1}{\rho R} \frac{\partial}{\partial R}\left(R \rho \overline{v_{\mathrm{R}} v_{\mathrm{z}}}\right)-\frac{1}{\rho} \frac{\partial}{\partial z}\left(\rho \overline{v_{\mathrm{z}}^{2}}\right) .
\end{aligned}
$$

We consider that there is no net streaming motion along radial and vertical directions in the Galaxy, hence we can write $\overline{v_{\mathrm{R}}^{2}}=\sigma_{\mathrm{R}}^{2}$ and $\overline{v_{\mathrm{z}}^{2}}=\sigma_{\mathrm{z}}^{2}$ where $\sigma_{\mathrm{R}}$ and $\sigma_{\mathrm{z}}$ represent velocity dispersions of stars along $R$ and $z$ directions respectively. $\overline{v_{\phi}^{2}}$ can be written as $\overline{v_{\phi}^{2}}=\sigma_{\phi}^{2}+{\overline{v_{\phi}}}^{2}$, where $\overline{v_{\phi}}$ represents observed mean rotation of stars in the disc and $\sigma_{\phi}$ is the azimuthal velocity dispersion.

At $z=0$, the mid-plane of the Galaxy, the velocity ellipsoid remains perfectly aligned with the cylindrical coordinate axes, which makes $\overline{v_{\mathrm{R}} v_{\mathrm{z}}}=0$ (Mihalas \& Routly 1968). But away from the mid-plane, i.e, for $z \neq 0$, the ellipsoid can be shown to be tilted with respect to the coordinates axes (Mihalas \& Routly 1968) and such a deviation gives rise to a non-zero $\overline{v_{\mathrm{R}} v_{\mathrm{z}}}$ term, as also found in observations (Introduction). Recently, such a tilted ellipsoid was included in the studies by Cappellari (2019), and Nitschai et al. (2019) who solved the Jeans equations to obtain the kinematical quantities and compared these with observations. These papers considered the tilted velocity ellipsoid which was taken to be aligned along the spherical system for $z \neq 0$. However since they solved the equations in spherical coordinates the cross term drops out of the calculation. We note that these papers did not solve the joint Poisson equation and the Jeans equations to obtain the vertical density distribution of stars as done in our work.

Here, we write the Jeans equations in cylindrical coordinates and consider the velocity ellipsoid to be aligned with spherical coordinates, centred at the centre of the Galaxy, at all radii for $z \neq 0$. Thus we include the effect of the cross term. We use the expression for $\overline{v_{\mathrm{R}} v_{\mathrm{z}}}$ given as $\left(\sigma_{\mathrm{R}}^{2}-\sigma_{\mathrm{z}}^{2}\right)(z / R)$ (Mihalas \& Routly 1968; Binney \& Tremaine 1987). Using equations (4) and (5), we calculate the radial and vertical terms of the Poisson equation to be:

$$
\begin{aligned}
\frac{1}{R} \frac{\partial}{\partial R}\left(R \frac{\partial \Phi}{\partial R}\right)= & -\frac{1}{R} \frac{\partial}{\partial R}\left[\frac{R}{\rho} \frac{\partial}{\partial R}\left(\rho \sigma_{R}^{2}\right)\right] \\
& -\frac{1}{R} \frac{\partial}{\partial R}\left[\frac{1}{\rho} \frac{\partial}{\partial z}\left(\rho z\left(\sigma_{R}^{2}-\sigma_{z}^{2}\right)\right)\right] \\
& -\frac{1}{R} \frac{\partial \sigma_{R}^{2}}{\partial R}+\frac{1}{R} \frac{\partial}{\partial R}\left(\sigma_{\phi}^{2}+{\overline{v_{\phi}}}^{2}\right) \\
\frac{\partial^{2} \Phi}{\partial z^{2}}= & -\frac{\partial}{\partial z}\left[\frac{1}{\rho R} \frac{\partial}{\partial R}\left(\rho z\left(\sigma_{R}^{2}-\sigma_{z}^{2}\right)\right)\right] \\
& -\frac{\partial}{\partial z}\left[\frac{1}{\rho} \frac{\partial}{\partial z}\left(\rho \sigma_{z}^{2}\right)\right]
\end{aligned}
$$

We consider an exponential stellar disc whose surface density falls off as $\Sigma=\Sigma_{0} \exp \left(-R / R_{\mathrm{D}}\right)$. Similarly, we also consider the radial velocity dispersion to fall off exponentially as $\sigma_{\mathrm{R}}=\sigma_{0} \exp (-$ $\left.R / R_{\mathrm{vel}}\right)$ and define the vertical and azimuthal dispersions in terms of ratio to $\sigma_{\mathrm{R}}$, e.g. $b_{\mathrm{z}}=\sigma_{\mathrm{z}}^{2} / \sigma_{\mathrm{R}}^{2}$ and $b_{\phi}=\sigma_{\phi}^{2} / \sigma_{\mathrm{R}}^{2}$.

Using the above physical assumptions, and expanding equations (6) and (7) and substituting into equation (3), we obtain the second-order differential equation describing the vertical density distribution as:

$$
\begin{aligned}
\frac{\mathrm{d}^{2} \rho}{\mathrm{d} z^{2}}= & \frac{-4 \pi G \rho^{2}}{\sigma_{\mathrm{z}}^{2}}+\frac{1}{\rho}\left(\frac{\mathrm{d} \rho}{\mathrm{d} z}\right)^{2}+\frac{\rho}{\sigma_{\mathrm{z}}^{2}} \frac{2 \overline{v_{\phi}}}{R}\left(\frac{\partial \overline{v_{\phi}}}{\partial R}\right) \\
& -\frac{z\left(1-b_{\mathrm{z}}\right)}{b_{\mathrm{z}} R}\left(\frac{2}{R_{\mathrm{D}}}-\frac{2}{R_{\mathrm{vel}}}\right) \frac{\mathrm{d} \rho}{\mathrm{d} z} \\
& +\frac{\rho}{b_{\mathrm{z}}}\left[\frac{2}{R R_{\mathrm{vel}}}-\frac{4}{\left(R_{\mathrm{vel}}\right)^{2}}+\frac{1}{R R_{\mathrm{D}}}-\frac{2}{R_{\mathrm{D}} R_{\mathrm{vel}}}+\frac{4\left(1-b_{\mathrm{z}}\right)}{R R_{\mathrm{vel}}}\right. \\
& \left.+\frac{2\left(1-b_{\phi}\right)}{R R_{\mathrm{vel}}}+\frac{\left(1-b_{\mathrm{z}}\right)}{R R_{\mathrm{D}}}\right]
\end{aligned}
$$


The first three terms of the above equation correspond to equation (1) and hence the rest of the terms in equation (8) arise due to the various generalizations considered here. It allows us to study the effect of any kinematical term in a methodical way. The algebraic simplifications leading to the above equation consider $(1 / \rho)(\partial \rho / \partial R)=-1 / R_{\mathrm{D}}$ which assumes that $\rho=\Sigma / 2 h_{\mathrm{z}}$, where $h_{\mathrm{z}}$ is the disc thickness at any radius. Though the disc thickness does not remain constant with radius (as shown later in Section 3.2), we expect that this approximation is justified as it will give rise to only second-order effects in the results at a particular radius.

Equation (8) represents a general formalism and therefore can be applied for any typical disc galaxy satisfying the above physical assumptions. Here, we have applied it for our Galaxy and we describe all the required input parameters in the following subsection.

\subsection{Input parameters and numerical solution}

For the surface density of the stellar disc of the Milky Way, we have used $R_{\mathrm{D}}=3.2 \mathrm{kpc}$ and $\Sigma_{0}=640.9 \mathrm{M}_{\odot} \mathrm{pc}^{-2}$ (Mera, Chabrier \& Schaeffer 1998). For $\sigma_{\mathrm{R}}$, we consider $R_{\mathrm{vel}}=8.7 \mathrm{kpc}$ and $\sigma_{0}=$ $105.0 \mathrm{~km} \mathrm{~s}^{-1}$ as observed by Lewis \& Freeman (1989). We take $b_{\mathrm{z}}$ to be $(0.45)^{2}$, consistent with the observation in the solar neighbourhood (Dehnen \& Binney 1998; Mignard 2000) and assume this to be valid at all radii. These same input parameter values were used for the $\operatorname{sech}^{2}$ model (Section 1). The azimuthal dispersion is related to radial dispersion as in epicyclic approximation, hence the ratio $b_{\phi}$ is calculated to be:

$b_{\phi}=\frac{\sigma_{\phi}^{2}}{\sigma_{\mathrm{R}}^{2}}=\frac{1}{2} \frac{\left(V_{\mathrm{c}} / R+\mathrm{d} V_{\mathrm{c}} / \mathrm{d} R\right)}{V_{\mathrm{c}} / R}$

where $V_{\mathrm{c}}$ is the circular velocity. We note that this expression is derived considering a circular orbit for stars and hence a true circular velocity curve should be used. But here we consider the mean rotation velocity $\overline{v_{\phi}}$ from the observed rotation curve, which deviates from the true circular velocity, to calculate the ratio. We expect that this approximation is justified, as it may lead to only second-order effect in the $b_{\phi}$ values. We use the observed rotation velocity data from Sofue (2012) and fit a polynomial to the observed data. Using the best-fitting curve, we calculate both the rotation velocity and its gradient at any radius and determine the ratio $b_{\phi}$ using equation (9). The values obtained are $0.46,0.54,0.57,0.56,0.49$, and 0.41 at $R=2,3,4,5,6$, and $7 \mathrm{kpc}$ respectively. Starting from and beyond the solar neighbourhood, the observed data shows large fluctuations, however, the theoretically fitted smooth curve in Sofue (2012) is nearly flat. Therefore, for $R \geq 8.5 \mathrm{kpc}$, we consider the rotation curve to be flat for simplicity which gives $b_{\phi}$ identically $=0.5$ from equation (9).

We note that, as the velocity dispersion of stars falls with radius, its value may get less than the gas dispersion, beyond a certain radius. Since stars are formed from gas clouds, hence they cannot have a lower velocity dispersion than the gas itself, as explained in Sarkar \& Jog (2018). The HI velocity dispersion in the outer Galaxy is observed to saturate around $7 \mathrm{~km} \mathrm{~s}^{-1}$ (Kamphuis 1993; Dickey 1996). Therefore, we keep the stellar vertical and azimuthal dispersion values constant at $7.5 \mathrm{~km} \mathrm{~s}^{-1}$ from $R=18$ and $20 \mathrm{kpc}$ onward respectively to keep it higher than the gas dispersion and update the values of $b_{\phi}$ and $b_{z}$ accordingly at those radii.

We solve equation (8) using the method as in Narayan \& Jog (2002), and Sarkar \& Jog (2018). That is, we solve it by applying the fourth-order Runge-Kutta method, where the observed surface density of stars is used as one boundary condition and the second
Table 1. Results for the mid-plane density $\rho_{0}$ using the $\operatorname{sech}^{2}$ model, Model A (with only a non-flat, observed rotation curve), and Model B (the complete, general one).

\begin{tabular}{lccccc}
\hline $\begin{array}{l}\text { Radius } \\
(\mathrm{kpc})\end{array}$ & $\begin{array}{c}\left(\rho_{0}\right)_{\mathrm{sec}^{2}} \\
\left(M_{\odot} \mathrm{pc}^{-3}\right)\end{array}$ & $\begin{array}{c}\left(\rho_{0}\right)_{\text {model A }} \\
\left(M_{\odot} \mathrm{pc}^{-3}\right)\end{array}$ & $\begin{array}{c}\Delta_{\mathrm{A}} \\
(\%)\end{array}$ & $\begin{array}{c}\left(\rho_{0}\right)_{\text {model B }} \\
\left(M_{\odot} \mathrm{pc}^{-3}\right)\end{array}$ & $\begin{array}{c}\Delta_{\mathrm{B}} \\
(\%)\end{array}$ \\
\hline 2.0 & 0.568 & 0.604 & +6.3 & 0.463 & -18.5 \\
3.0 & 0.381 & 0.365 & -4.2 & 0.275 & -27.8 \\
4.0 & 0.258 & 0.236 & -8.5 & 0.190 & -26.4 \\
5.0 & 0.173 & 0.162 & -6.4 & 0.140 & -19.1 \\
6.0 & 0.117 & 0.118 & +0.85 & 0.108 & -7.7 \\
7.0 & 0.078 & 0.086 & +10.2 & 0.081 & +3.8 \\
8.5 & 0.043 & - & 0.0 & 0.041 & -4.6 \\
10.0 & 0.024 & - & 0.0 & 0.023 & -4.2 \\
12.0 & 0.011020 & - & 0.0 & 0.011045 & +0.2 \\
14.0 & $5.13 \times 10^{-3}$ & - & 0.0 & $5.27 \times 10^{-3}$ & +2.7 \\
16.0 & $2.38 \times 10^{-3}$ & - & 0.0 & $2.55 \times 10^{-3}$ & +7.1 \\
18.0 & $6.54 \times 10^{-4}$ & - & 0.0 & $8.12 \times 10^{-4}$ & +24.2 \\
20.0 & $2.17 \times 10^{-4}$ & - & 0.0 & $3.03 \times 10^{-4}$ & +39.6 \\
22.0 & $8.78 \times 10^{-5}$ & - & 0.0 & $1.24 \times 10^{-4}$ & +41.2 \\
\hline
\end{tabular}

condition is given by $\mathrm{d} \rho / \mathrm{d} z=0$, defined at $z=0$. This condition is satisfied for any realistic vertical density distribution which will be homogeneous close to the mid-plane.

\section{RESULTS: VERTICAL STELLAR DISC STRUCTURE}

\subsection{Effect on the mid-plane density $\rho_{0}$ of the stellar disc}

We solved for the vertical density distribution for two cases. First, when the disc structure is solved by writing the radial term in equation (3) in terms of the observed, non-flat rotation curve from $R=2$ to $7 \mathrm{kpc}$, to study its effect specifically. This is done by solving the following equation (Model A), as obtained in Sarkar \& Jog ( 2019):

$\frac{\mathrm{d}^{2} \rho}{\mathrm{d} z^{2}}=\frac{-4 \pi G \rho^{2}}{\sigma_{\mathrm{z}}^{2}}+\frac{1}{\rho}\left(\frac{\mathrm{d} \rho}{\mathrm{d} z}\right)^{2}+\frac{\rho}{\sigma_{\mathrm{z}}^{2}} \frac{2 \overline{v_{\phi}}}{R}\left(\frac{\partial \overline{v_{\phi}}}{\partial R}\right)$

The observed mean rotation velocity values (Sofue 2012) are used in the last term in the above equation.

The resulting mid-plane density $\rho_{0}$ values are listed in Table 1, under 'Model A' and are compared with the results from sech ${ }^{2}$ model (stars-alone case) obtained in Sarkar \& Jog (2018), with $\Delta_{\mathrm{A}}$ giving the percent difference between these two models. We note that $\rho_{0}$ has increased at some radii $(R=2,6$, and $7 \mathrm{kpc})$ and has decreased at some radii $(R=3,4$, and $5 \mathrm{kpc})$ which happens due to the negative and positive gradients, respectively, present in the non-flat rotation curve at the corresponding radii. The last term in equation (10), shows that the amount of change introduced in the $\left(\rho_{0}\right)$ values depends on both the gradient and the absolute value of the rotation velocity. Beyond $R=8.5 \mathrm{kpc}$, Model A coincides with the $\operatorname{sech}^{2}$ model, hence the density values are the same and difference is 0 per cent.(Table 1$)$.

Second, in Model B we consider the effect of $R-z$ coupling, a non-flat rotation curve and planar random motions, hence we term it as the most general and the complete one. For this case, we solve equation (8) from $R=2$ to $22 \mathrm{kpc}$, and compare the results with those from the $\operatorname{sech}^{2}$ model (Table 1) with $\Delta_{\mathrm{B}}$ denoting the per cent difference between these two models. We note that up to $R=10 \mathrm{kpc}$, $\rho_{0}$ is lower than that of $\operatorname{sech}^{2}$ model, except at $R=7 \mathrm{kpc}$ where 

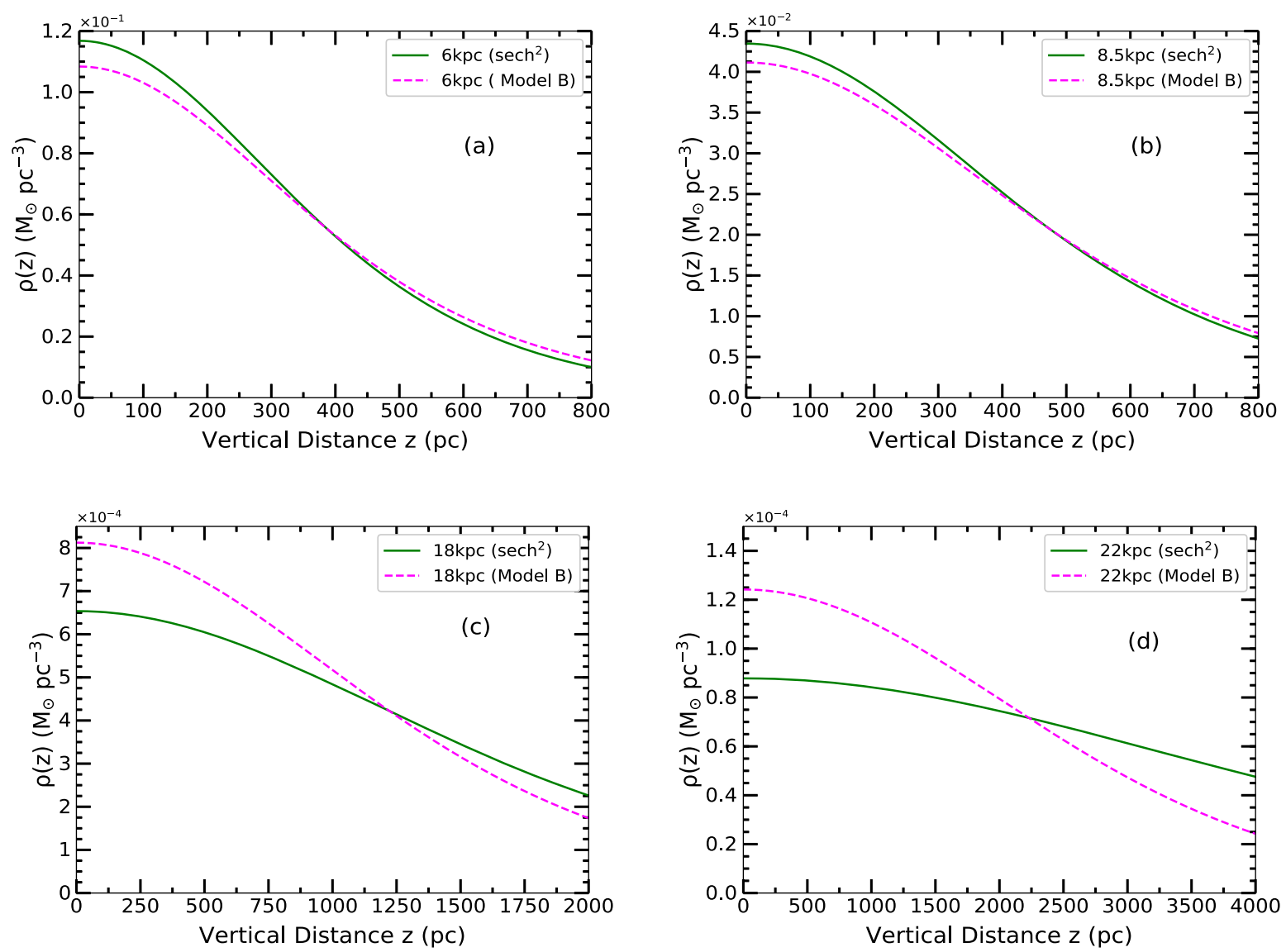

Figure 1. Plot of vertical density distribution $(\rho(z))$ of stars at $R=6,8.5,18$, and $22 \mathrm{kpc}$ (a)-(d) respectively. The green solid curves represent $\rho(z)$ for a $\mathrm{sech}^{2}$ distribution (see equation 2 in Introduction) and the magenta dashed curves represent $\rho(z)$ obtained using the complete, general model (Model B) here. The difference between these two distributions is more prominent in the outer Galaxy and turns out to be very small at the 6 kpc and solar radius. This shows the significance of using the complete Poisson and Jeans equations while solving for $\rho(z)$ for stars, specially in the Outer Galaxy.

the density is higher as a result of the high magnitude of negative gradient present in the rotation curve at that radius. We find that the magnitude of change in $\rho_{0}$ is higher in the inner radii and lower toward the solar neighbourhood region. In contrast, the resulting values of the mid-plane density are higher than those from the sech ${ }^{2}$ model at radii beyond $R=12 \mathrm{kpc}$. This change increases with radius and gets very prominent, more than $\sim 30$ per cent, in the outer disc region, beyond $R=18 \mathrm{kpc}$. This may be explained as follows. We expect that in the outer disc region, where the surface density is low and the vertical distribution becomes an extended one, as found in Sarkar \& Jog ( 2018), the contribution of the radial gradient of potential may not be negligible compared to the vertical gradient in the Poisson equation. Further, the effect of $R-z$ coupling and the planar random motions can have substantial effect on the vertical density distribution since the self-gravity of the disc becomes low. Therefore, in the outer disc region it becomes necessary to consider the full Poisson equation with the full Jeans equations as done here.

For the intermediate radial range from 7 to $12 \mathrm{kpc}$, the values of change are small (a few per cent), and at $R=12 \mathrm{kpc}$, the change is the lowest, almost zero. This is by sheer coincidence of the various input parameters which allow for the $\operatorname{sech}^{2}$ model to be valid in the intermediate radial range. Hence a simple $\operatorname{sech}^{2}$ model in the solar neighbourhood assumed so far in the literature has been well justified. We note that this range showing small change may vary slightly depending on the actual values of the input parameters used. The changes shown in this range (see $\Delta B$ values in Table 1) do not show a clear pattern with radius. We note that it is the complex interplay among the various kinematical terms present in the vertical and radial terms in the Poisson equation (in equation 8) that sets the value of the mid-plane density and hence the difference with $\mathrm{sech}^{2}$ model results, including the sign of the difference. Beyond $R=7 \mathrm{kpc}$, the rotation curve is taken to be flat so the difference then is purely due to the kinematical terms involving the random motions and the cross term.

We have shown the plots for $\rho(z)$ versus $z$ for radii $6,8.5,18$, and $22 \mathrm{kpc}$ in Fig. 1 from Model B and the sech ${ }^{2}$ model. We note that we have provided the $\rho_{0}$ values with higher decimal accuracy to facilitate comparison among various models, though the observed data are not known to this accuracy. Interestingly, we find that on inclusion of these kinematical terms, the resulting $\rho(z)$ profiles are found to fit to a function of type $\operatorname{sech}^{2 / n}$ with $n$ varying with radius (as was also found for the case of multicomponent model shown in Sarkar \& Jog (2018) - which did not consider these kinematical effects), instead of the typical of $\operatorname{sech}^{2}$ function.

\subsection{Effect on the scale height (HWHM) of the stellar disc}

We measure the disc scale height in terms of the HWHM of the vertical density distribution and list the values in Table 2 . The 
Table 2. Results for HWHM using the $\operatorname{sech}^{2}$ model, model with only a nonflat, observed rotation curve (Model A) and the complete, general model (Model B).

\begin{tabular}{lccccc}
\hline $\begin{array}{l}\text { Radius } \\
(\mathrm{kpc})\end{array}$ & $\begin{array}{c}(\mathrm{HWHM})_{\text {sech }^{2}} \\
(\mathrm{pc})\end{array}$ & $\begin{array}{c}(\mathrm{HWHM})_{\mathrm{A}} \\
(\mathrm{pc})\end{array}$ & $\begin{array}{c}\Delta_{\mathrm{A}} \\
(\%)\end{array}$ & $\begin{array}{c}(\mathrm{HWHM})_{\mathrm{B}} \\
(\mathrm{pc})\end{array}$ & $\begin{array}{c}\Delta_{\mathrm{B}} \\
(\%)\end{array}$ \\
\hline 2.0 & 266.3 & 251.8 & -5.4 & 311.9 & +17.1 \\
3.0 & 290.2 & 301.8 & +4.0 & 377.5 & +30.0 \\
4.0 & 313.6 & 340.0 & +8.4 & 403.3 & +28.6 \\
5.0 & 341.9 & 362.1 & +5.9 & 408.8 & +19.6 \\
6.0 & 370.8 & 366.4 & -1.2 & 393.3 & +6.1 \\
7.0 & 405.1 & 371.7 & -8.2 & 389.0 & -4.0 \\
8.5 & 456.7 & - & 0.0 & 467.4 & +2.3 \\
10.0 & 515.4 & - & 0.0 & 527.3 & +2.3 \\
12.0 & 606.2 & - & 0.0 & 599.4 & -1.1 \\
14.0 & 708.3 & - & 0.0 & 663.6 & -6.3 \\
16.0 & 822.2 & - & 0.0 & 760.5 & -7.5 \\
18.0 & 1568.9 & - & 0.0 & 1260.7 & -19.6 \\
20.0 & 2721.8 & - & 0.0 & 1858.9 & -31.7 \\
22.0 & 4279.9 & - & 0.0 & 2515.3 & -41.2 \\
\hline
\end{tabular}

symbols $\Delta_{\mathrm{A}}$ and $\Delta_{\mathrm{B}}$ have the same meaning as in Table 1. In Model $\mathrm{A}$, when the gradient in the rotation curve is positive it makes the disc puff up, and when the gradient is negative it decreases the disc thickness compared to that of $\operatorname{sech}^{2}$ model due to higher mid-plane density (as similar to the case for UGC 7321, Sarkar \& Jog 2019). From $R=8.5 \mathrm{kpc}$, Model A coincides with the $\operatorname{sech}^{2}$ model, hence the difference is 0 per cent(Table 2).

In Model B, the change in HWHM from that of the $\operatorname{sech}^{2}$ model is positive upto $R=10 \mathrm{kpc}$, except at $R=7 \mathrm{kpc}$, due to the high negative gradient present in the rotation curve, as discussed earlier in Section 3.1. We get negative changes beyond $R=12 \mathrm{kpc}$ and also note that the amount of change is again the least at $R=12 \mathrm{kpc}$. The change in scale height value becomes as high as 30-40 per cent in the outer disc region. We note that the corresponding changes in $\rho_{0}$ and HWHM are compatible with each other in both the models.

Our results show that as in the $\operatorname{sech}^{2}$ case, in the complete model (Model B) also, the vertical disc thickness increases with radius and gives rise to flaring in the outer Galaxy. This again shows (as in Sarkar \& Jog 2018, 2019 where the kinematical effects were not included) that flaring in the outer disc region is a generic phenomenon. We note that the absolute magnitude of flaring from $R=2$ to $22 \mathrm{kpc}$ decreases by a factor of two (from 16 to 8 ) compared to the $\operatorname{sech}^{2}$ model. This shows the importance of considering various effects (as in equation 8) while studying the vertical structure.

\subsection{Addition of dark matter halo gravity in the outer Galaxy}

For a realistic multicomponent, gravitationally coupled galactic disc (but without the kinematical effects included here), the vertical distribution of stars is shown to be constrained by gas and dark matter halo gravity in the inner and the outer discs, respectively (Sarkar \& Jog 2018). Here, we add the halo gravity to Model B at $R=18$ and $22 \mathrm{kpc}$, considering the disc to be effectively a gravitationally coupled stars plus halo system and solve for $\rho(z)$. The $\rho_{0}$ values increase by a factor of 2.3 at $R=18 \mathrm{kpc}$ and a factor of 3 at $R=22 \mathrm{kpc}$, compared to the stars-alone disc, and much higher compared to the kinematical effects alone (Table 1). The HWHM values also get lowered correspondingly, by a factor of 2.2 and 3.1, respectively and this tends to decrease the flaring. We do not add the gas gravity in the equation ( 8) to solve it in the inner Galaxy, as the modified equation will contain second-order variation in gas surface density, which is not known to us from observations.

\section{CONCLUSIONS}

We have studied theoretically the self-consistent vertical structure of a galactic stellar disc using the most general and complete model (Model B here) which considers the complete axisymmetric Poisson equation containing both the radial and vertical terms. These are calculated using the complete Jeans equations which consider a non-flat rotation curve, planar random motions, and a tilted velocity ellipsoid. Thus, in this treatment the $R$ and $z$ motions are taken to be coupled. We found that the mid-plane density $\left(\rho_{0}\right)$ is lower than that from the typical $\mathrm{sech}^{2}$ model at the inner radii and becomes higher from $R=12 \mathrm{kpc}$ onward. The HWHM values also change accordingly,i.e. higher at the inner radii and lower from $R=12 \mathrm{kpc}$. Interestingly, by sheer chance, for the observed input parameters in the small radial range in the solar neighbourhood $\sim 7-12 \mathrm{kpc}$, the changes are very small (<a few per cent), whereas in outer disc region they are very prominent ( $\sim 40$ per cent). This is due to the low density and hence low self-gravity and extended vertical disc distribution in the outer Galaxy.

Thus our work shows that the standard isothermal model resulting in a sech ${ }^{2}$ vertical density distribution (Spitzer 1942) for a thin, selfgravitating galactic disc turns out to be well justified in the solar neighbourhood, but not in the outer disc. Hence, one must include all the terms as discussed above to get the correct vertical distribution in the following three general cases of low-density region, namely in the outer Galaxy, at high $z$ and for a thick disc. The predicted changes due to the various kinematic effects studied here can now be verified with the new, accurate data for example from Gaia or LAMOST.

\section{ACKNOWLEDGEMENTS}

We thank the anonymous referee for constructive and helpful comments. SS thanks CSIR for a fellowship, and CJ thanks the DST, Government of India for support via J.C. Bose fellowship (SB/S2/JCB-31/2014).

\section{REFERENCES}

Bahcall J., 1984, ApJ, 276, 156

Banerjee A., Jog C. J., Brinks E., Bagetakos I., 2011, MNRAS, 415, 687

Binney J., Tremaine S., 1987, Galactic Dynamics, Princeton Univ. Press, Princeton, NJ

Bovy J., Tremaine S., 2012, ApJ, 756, 89

Cappellari M., 2008, MNRAS, 390, 71

Cappellari M., 2019, preprint(arXiv:1907.09894)

Dehnen W., Binney J., 1998, MNRAS, 298, 387

Dickey J. M., 1996, in Blitz L., Teuben P., eds, Unsolved Problems of the Milky Way, Vol. 169, IAU Symp., Kluwer, Dordrecht, p. 489

Everall A., Evans N. W., Belokurov V., Schönrich R., 2019, MNRAS, 489, 910

Hagen J. H. J., Helmi A., 2018, A\&A, 615, A99

Hagen J. H. J., Helmi A., de Zeeuw P. T., Posti L., 2019, A\&A, 629, A70

Kamphuis J. J., 1993, PhD thesis, University of Groningen

Lewis J. R., Freeman K. C., 1989, AJ, 97, 139

Mera D., Chabrier G., Schaeffer R., 1998, A\&A, 330, 953

Mignard F., 2000, A\&A, 354, 522 
Mihalas D., Routly P. M., 1968, Galactic Astronomy, W. H. Freeman And Company, San Francisco, London

Narayan C. A., Jog C. J., 2002, A\&A, 394, 89

Narayan C. A., Saha K., Jog C. J., 2005, A\&A, 440, 523

Nitschai M. S., Cappellari M., Neumayer N., 2019, preprint (arXiv:1909.0 5269v1)

Oort J. H., 1932, BAN, 6, 349

Sánchez-Salcedo F. J., Flynn C., de Diego J. A., 2016, ApJ, 817, 13
Sarkar S., Jog C. J., 2018, A\&A, 617, A142

Sarkar S., Jog C. J., 2019, A\&A, 628, A58

Sofue Y., 2012, PASJ, 64, 75

Spitzer L., 1942, ApJ, 95, 329

Wegg C., Gerhard O., Bieth M., 2019, MNRAS, 485, 3296

This paper has been typeset from a $\mathrm{T}_{\mathrm{E}} \mathrm{X} / \mathrm{LT}_{\mathrm{E}} \mathrm{X}$ file prepared by the author. 